\title{
Functional MRI language mapping in pre-surgical epilepsy patients: Findings from a series of patients in the Epilepsy Unit at Mediclinic Constantiaberg
}

\author{
V L Ives-Deliperi, ${ }^{1} \mathrm{PhD} ; \mathbf{J}$ T Butler, ${ }^{2,3} \mathrm{MB}$ ChB, FCP; E M Meintjes, ${ }^{1} \mathrm{PhD}$ \\ ${ }^{1}$ MRC/UCT Medical Imaging Research Unit, Department of Human Biology, University of Cape Town, South Africa \\ ${ }^{2}$ Department of Neurology, Stellenbosch University, South Africa \\ ${ }^{3}$ Department of Neurology, University of Cape Town, South Africa
}

Corresponding author: V L Ives-Deliperi (vives@mweb.co.za)

\begin{abstract}
Background. Functional magnetic resonance imaging (fMRI) is commonly applied to study the neural substrates of language in clinical research and for neurosurgical planning. fMRI language mapping is used to assess language lateralisation, or determine hemispheric dominance, and to localise regions of the brain involved in language. Routine fMRI has been introduced in the Epilepsy Unit at Mediclinic Constantiaberg to contribute to the current functional mapping procedures used in pre-surgical planning.

Method. In this paper we describe the language paradigms used in these routine studies as well as the results from 22 consecutive epilepsy patients. Multi-subject analyses were performed to assess the reliability of activation patterns generated by two language mapping paradigms, namely a verb generation task and passive listening task. Results from a finger-tapping task are also presented.

Results. The paradigms generate reliable and robust signal changes, enabling both the lateralisation of language and localisation of expressive and receptive language cortex.

Conclusion. The fMRI results are meaningful at the group and individual level and can be recommended for language mapping in presurgical patients.
\end{abstract}

S Afr Med J 2013;103(8):563-567.DOI:10.7196/SAMJ.6336

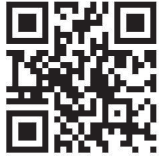

The surgical treatment of medically intractable epilepsy is effective in controlling seizures. ${ }^{[1,2]}$ Language mapping is a critical part of the pre-surgical evaluation of patients to determine hemispheric dominance and identify areas involved in language. Localising eloquent regions means that they may be preserved in surgery to avoid post-operative aphasia, while maximising the area of resection to obtain the optimal seizure outcome.

Traditional methods used to lateralise language function include neuropsychological testing and Wada testing. The Wada test, or intra-carotid amytal procedure (IAP), has been considered the gold standard for lateralising language. ${ }^{[3]}$ The procedure involves injecting a barbiturate into the carotid artery to anaesthetise one hemisphere at a time, during which memory and language tests are conducted to assess the functional reserve of the contralateral hemisphere. Although the test has been shown to be reliable, it is invasive, costly, time-consuming and carries a risk of stroke and death. ${ }^{[4]}$ In recent years functional magnetic resonance imaging (fMRI) has been increasingly applied as an alternative method of determining hemispheric dominance, since it is safer and more costeffective and generates a continuous measure of language. Results of language fMRI and the IAP have been concordant in a large majority of individuals with epilepsy. ${ }^{[5-7]}$ In a meta-analysis of 23 studies including 442 epilepsy patients, the sensitivity and specificity of fMRI for atypical language dominance, compared with the Wada test, were $83.5 \%$ and $88 \%$ respectively. ${ }^{[8]}$

The task of localising cortical areas critical to language is more challenging. Electrocortical stimulation (ECS) remains the most reliable technique, even with the advancements in fMRI. In ECS electrical current is applied to the cortex, either via subdural electrodes extra-operatively, or directly to the cortex intra-operatively. Electrical current inhibits neuronal activity in the language cortex. Clinical tests are conducted during the application of electrical current to assess the effects of loss of function in the cortical region stimulated. It is generally agreed that while fMRI identifies cortical regions involved in language, ECS identifies those critical to the function. ${ }^{[9]}$

fMRI language mapping in epilepsy patients relies on blood oxygen level-dependent (BOLD) contrast. This contrast measures changes in haemodynamic response related to neuronal energy consumption on $\mathrm{T} 2^{\star}$-weighted MR images while an expressive or receptive language task is being performed. ${ }^{[10]}$ Functional paradigms are designed as either blocked or event-related tasks. Blocked paradigms consist of a series of alternating active and passive conditions and the method is statistically robust since the signal acquired for each condition is high. Using a general linear model, regions are identified where changes in the $\mathrm{T} 2^{*}$-weighted signal are temporally correlated with the active and passive conditions of the task. Active and passive conditions are contrasted in the analysis, to identify the regions that demonstrate greater activity during active conditions than passive ones. The identified regions are visualised on a T1-weighted MR image and interpreted as being involved in the function performed during the task. Once activation maps have been produced, hemispheric dominance is assessed by either counting the active voxels (volumetric pixels representing a value in 3-dimensional space) at a specified threshold in each hemisphere, or assessing BOLD signal changes in pre-defined anatomical regions. Findings from threshold-independent measures have been shown to correlate most strongly with other lateralisation techniques, such as the Wada test. ${ }^{[1,12]}$

Language is a complex task relying on a number of discrete but interacting cognitive processes that recruit a distributed network of brain regions. Therefore, it is recommended that multiple language tasks be administered in fMRI in order to calculate language 
dominance as a continuous variable. ${ }^{[13]}$ In localising language function, several tasks have been shown to reliably activate expressive and receptive language cortex (Table 1). Generally, verbal fluency paradigms require expressive language and secondarily, language comprehension and therefore activations are noted in Broca's areas and Wernicke's area in the dominant hemisphere, in addition to pre-motor cortex, posterior fusiform gyrus, middle temporal gyrus and dorsolateral prefrontal cortex. ${ }^{[1]}$ Paradigms of passive listening have been shown to reliably activate receptive cortex. ${ }^{[1]}$ Receptive language paradigms commonly show bilateral activation in the posterior superior temporal gyri. ${ }^{[14]}$

Routine fMRI of language in pre-surgical epilepsy patients has been introduced in the Epilepsy Unit at Mediclinic Constantiaberg. These studies contribute to the broader functional mapping protocol and are used to guide ECS and assist in surgical planning. Several language tasks were tested in a series of 6 epilepsy patients and 2 healthy controls prior to finalising the routine language protocol, to identify the tasks that most reliably produced signal changes in eloquent cortex. Theses tasks included counting, verbal fluency, verb generation and passive listening and were designed in accordance with how they are commonly used in other centres. ${ }^{[14-16]}$ The verb generation and passive listening tasks produced the most robust and reliable signal changes in these studies. The findings from a series of subsequent language fMRI studies are presented in Table 1.

\section{Methods}

fMRI results from one motor-mapping task and two language-mapping tasks in 22 consecutive patients were included in multi-subject analyses. Results of the finger-tapping task have been included here (Table 3 and Fig. 2) to demonstrate the validity of the blocked paradigm and the satisfactory participation of the patients, since BOLD signal changes during motor tasks are highly reliable and robust. In addition, active participation in the task can be directly observed, unlike in language tasks. Each patient performed these tasks in single scanning sessions. This is a retrospective analysis of clinical data, collected at our centre as part of the routine pre-surgical evaluation of patients with epilepsy. Patient demographics are outlined in Table 2.

\section{Procedures}

Routine fMRI was conducted according to a standard procedure. Before their imaging sessions patients were given an information sheet, describing the procedure and the tasks to be administered. Patients were briefed again on arrival at the imaging centre, the tasks were rehearsed and patients were given an opportunity to raise queries. All patients completed the Edinburgh Handedness Inventory $(\mathrm{EHI})^{[17]}$ as a self-reported measure of hemispheric dominance. All tasks were designed in blocked sequences and administered via audio facilities, with patients wearing the standard MR headphones.

\section{Functional paradigm}

The three tasks share a common blocked design, modelled on previously described functional imaging paradigms ${ }^{[11]}$ (see Fig. 1). Each task began with a $20 \mathrm{~s}$ rest period followed by 10 alternating rest and active $20 \mathrm{~s}$ blocks to total 3:40 minutes. In the active condition of the finger tapping task, patients were signalled, with the word 'tap', to tap the fingers of a given hand until instructed to stop. Patients then

\begin{tabular}{|c|c|c|c|c|}
\hline No. & Sex & Age & Handed (EHS) & Diagnosis \\
\hline 1 & M & 46 & RH (0.5) & LH TLE \\
\hline 2 & F & 33 & RH (0.8) & $\begin{array}{l}\text { LH FLE venous } \\
\text { fistula }\end{array}$ \\
\hline 3 & M & 58 & LH $(-0.3)$ & LH TLE \\
\hline 4 & M & 24 & RH (0.7) & LH TLE + HS \\
\hline 5 & M & 53 & $\mathrm{RH}(1)$ & $\begin{array}{l}\text { RH FLE } \\
\text { ganglioglioma }\end{array}$ \\
\hline 6 & M & 37 & LH (0.4) & $\begin{array}{l}\text { RH FLE } \\
\text { porencephalic cyst }\end{array}$ \\
\hline 7 & $\mathrm{~F}$ & 45 & $\mathrm{RH}(1)$ & LH TLE \\
\hline 8 & $\mathrm{~F}$ & 39 & RH (0.8) & RH TLE + HS \\
\hline 9 & M & 21 & RH (0.8) & RH TLE + HS \\
\hline 10 & $\mathrm{~F}$ & 39 & $\mathrm{RH}(1)$ & RH TLE + HS \\
\hline 11 & $\mathrm{~F}$ & 29 & $\mathrm{RH}(1)$ & LH TLE \\
\hline 12 & M & 11 & RH (0.8) & $\begin{array}{l}\text { LH cortical } \\
\text { malformation }\end{array}$ \\
\hline 13 & $\mathrm{~F}$ & 27 & $\mathrm{RH}(1)$ & RH TLE + HS \\
\hline 14 & $\mathrm{~F}$ & 21 & RH (0.7) & $\begin{array}{l}\text { RH porencephalic } \\
\text { cyst }\end{array}$ \\
\hline 15 & M & 35 & $\mathrm{RH}(1)$ & LH TLE \\
\hline 16 & $\mathrm{~F}$ & 25 & RH (0.6) & $\begin{array}{l}\text { RH cortical } \\
\text { malformation }\end{array}$ \\
\hline 17 & M & 28 & RH (0.8) & RH FLE \\
\hline 18 & $\mathrm{~F}$ & 37 & RH (0.7) & RH TLE + HS \\
\hline 19 & M & 37 & $\mathrm{RH}(1)$ & LH TLE \\
\hline 20 & $\mathrm{~F}$ & 43 & $\mathrm{RH}(1)$ & LH TLE \\
\hline 21 & $\mathrm{~F}$ & 27 & RH (0.6) & LH TLE \\
\hline 22 & F & 35 & RH (0.7) & LH TL/OL \\
\hline
\end{tabular}

$\mathrm{LH}=$ left hemisphere; $\mathrm{RH}=$ right hemisphere; TLE = temporal lobe epilepsy; HS = hippocampal sclerosis; $\mathrm{FLE}=$ frontal lobe epilepsy; $\mathrm{TL}=$ temporal lobe; $\mathrm{OL}=$ occipital lobe

Table 1. Language paradigms commonly used in fMRI

\begin{tabular}{lll}
\hline Paradigm & Task description & Activated regions \\
\hline Spontaneous speech & Counting & Expressive cortex \\
Verbal fluency & Generating words beginning with a given letter & Expressive and receptive cortex \\
Verb generation & Generating verbs from given nouns & Expressive and receptive cortex \\
Sentence generation & Describing a visual scene & Expressive cortex \\
Passive listening & Listening to a story & Receptive cortex \\
Repetition & Repeating words and phrases & Predominantly expressive cortex \\
fMRI = functional magnetic resonance imaging. & &
\end{tabular}


rested the hand in the control condition. In the active condition of the verb generation task, nouns were presented at $3 \mathrm{~s}$ intervals $(7 \mathrm{per}$ block) and the patients were required to think of a verb semantically related to the noun (i.e. 'what to do with') (see Fig. 3). Patients were instructed to generate the verb silently and not to mouth the word or speak, in order to restrict motion artifact. Stimuli in the control condition consisted of high and low tones to engage auditory processing and attention. Patients were instructed to listen to the tones attentively. In the active condition of the passive listening task, patients listened to a story being read by the administrator (see Fig. 4). Stimuli in the control condition consisted of high and low tones to engage auditory processing and attention. The patients were instructed to listen to the tones attentively.

\section{fMRI data acquisition}

Scans were acquired using a $1.5 \mathrm{~T}$ Avanto MRI scanner (Siemens Medical Systems, Erlangen, Germany). High-resolution anatomical images were acquired in the sagittal plane using a three-dimensional inversion recovery gradient echo sequence ( 176 slices, $\mathrm{TR}=2000 \mathrm{~ms}$, $\mathrm{TE}=50 \mathrm{~ms}, \mathrm{TI}=1100 \mathrm{~ms}$, resolution $1 \times 1 \times 1 \mathrm{~mm}^{3}, 256 \mathrm{~mm}$ FOV) . During the fMRI protocol 110 functional volumes sensitive to blood oxygen level-dependent contrast were acquired in all mapping tasks with a $\mathrm{T} 2{ }^{*}$-weighted gradient echo, echo planar imaging sequence $(\mathrm{TR}=2000 \mathrm{~ms}, \mathrm{TE}=50 \mathrm{~ms}, 21$ interleaved slices, $5 \mathrm{~mm}$ thick, gap $1.25 \mathrm{~mm}$, matrix size $64 \times 64$, resolution $3.4 \times 3.4 \times 3.4 \mathrm{~mm}^{3}$ ). In the tasks each of the 11 blocks lasted $20 \mathrm{~s}$ for a total scanning time of 3:40 minutes.

\section{fMRI data analysis}

All fMRI analyses were performed using Brain Voyager QX (Brain Innovation, Maastricht, The Netherlands). Two dummy images were excluded from analysis in each task run. Images were motioncorrected relative to the first volume with trilinear estimation and interpolation. Images were corrected for different slice acquisition times and linear trends, spatially smoothed using a Gaussian filter (FWHM $4 \mathrm{~mm}$ ), and temporally smoothed with a high pass filter of 3 cycles/point. Data sets exceeding movement criteria $3 \mathrm{~mm}$ displacement $/ 3.0^{\circ}$ rotation within a functional run were rejected. Each subject's functional data were co-registered to his/her highresolution anatomical MRI, rotated into the AC-PC plane and normalised to Talairach space using a linear transformation calculated on the anatomical images. The $3.4 \times 3.4 \times 3.4 \mathrm{~mm}^{3} \mathrm{fMRI}$ voxels were interpolated during Talairach normalisation to $3 \times 3 \times 3 \mathrm{~mm}^{3}$.

Whole-brain group analysis. For each task, a fixed-effect analysis of variance was performed using the general linear model with three predictors, one for the first $20 \mathrm{~s}$ rest, one for the control state and one for the active state (finger tapping, verb generation and listening) convolved by the standard haemodynamic function. Activations during the active conditions were examined by comparing activations during the active and control blocks. The voxel-wise threshold was set to $p<0.001$ (voxel-wise Bonferroni corrected for whole-brain multiple comparisons, min $t$ statistic 7.6). Clusters were reported if their extent was greater than 20 contiguous voxels, where the voxel size refers to the $1 \times 1 \times 1 \mathrm{~mm}^{3}$ resolution of the iso-voxelled structural images.

\section{Results}

The whole-brain group analysis of the finger-tapping task, conducted using the right hand in all participants, produced significant signal increases in the expected parts of the cortex representing contralateral primary motor cortex $t(9)=14.24, p<0.0001$, and ipsilateral cerebellum $t(9)=13.11, p<0.0001$.

In the verb generation task, signal increases were noted in anatomically typical expressive language cortex (Broca's area) in the left posterior inferior frontal gyrus $t(9)=7.86, p<0.0001$ and in receptive language cortex (Wernicke's area) in the left superior temporal gyrus $t(9)=5.34, p<0.0001$. The passive listening task

\begin{tabular}{|c|c|c|c|c|c|c|c|c|c|c|}
\hline \multirow[b]{2}{*}{ Rest } & \multicolumn{2}{|c|}{ Active } & \multicolumn{2}{|l|}{ Active } & \multicolumn{2}{|c|}{ Active } & \multicolumn{2}{|l|}{ Active } & \multicolumn{2}{|c|}{ Active } \\
\hline & & Cont. & & Cont. & & Cont. & & Cont. & & Cont. \\
\hline $20 \mathrm{~s}$ & $20 \mathrm{~s}$ & $20 \mathrm{~s}$ & $20 \mathrm{~s}$ & $20 \mathrm{~s}$ & $20 \mathrm{~s}$ & $20 \mathrm{~s}$ & $20 \mathrm{~s}$ & $20 \mathrm{~s}$ & $20 \mathrm{~s}$ & $20 \mathrm{~s}$ \\
\hline
\end{tabular}

Fig. 1. Functional mapping blocked paradigm.

Table 3. BOLD signal changes

\begin{tabular}{|c|c|c|c|c|c|c|}
\hline \multirow[b]{2}{*}{ Brain region } & \multirow[b]{2}{*}{ BA } & \multirow[b]{2}{*}{ Voxels } & \multicolumn{3}{|c|}{ Talairach co-ordinates } & \multirow[b]{2}{*}{ Voxel T } \\
\hline & & & $\mathbf{X}$ & $\mathbf{Y}$ & $\mathrm{Z}$ & \\
\hline \multicolumn{7}{|c|}{ Finger tapping (right-hand) } \\
\hline LH motor cortex & BA 4 & 5465 & -43 & -35 & -21 & 14.24 \\
\hline RH cerebellum & - & 3105 & 14 & -56 & 39 & 13.11 \\
\hline \multicolumn{7}{|l|}{ Verb generation } \\
\hline LH Broca’s area & BA 44 & 4170 & -49 & 16 & -3 & 7.86 \\
\hline LH Wernicke's area & BA 22 & 3097 & -58 & -50 & 21 & 5.34 \\
\hline \multicolumn{7}{|l|}{ Passive listening } \\
\hline LH Wernicke's area & BA 22 & 5187 & -64 & -35 & 2 & 7.23 \\
\hline RH Wernicke's area & BA 22 & 2776 & 56 & -26 & -6 & 5.89 \\
\hline
\end{tabular}




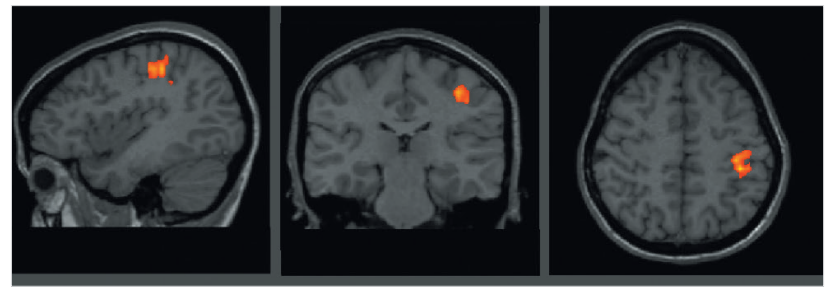

Fig. 2. BOLD signal increase in the left primary motor cortex produced in the group analysis of the finger tapping task (right hand).
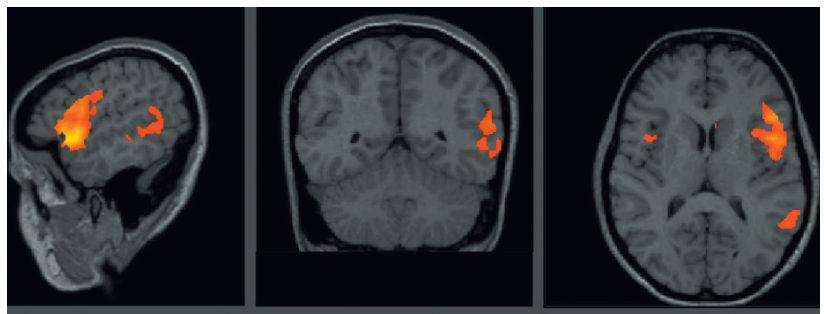

Fig. 3. BOLD signal increase produced in the group analysis of the verb generation task showing activation in left posterior inferior frontal gyrus (Broca's area) and left superior temporal gyrus (Wernicke's area).
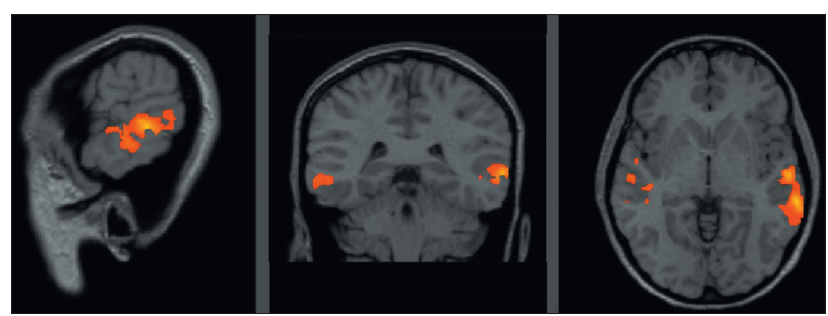

Fig. 4. BOLD signal increases produced in the group analysis of the passive listening task in the superior temporal lobes bilaterally (Wernicke's area).

produced signal increases in the superior temporal gyri of the left hemisphere $t(9)=7.23, p<0.0001$, and the right hemisphere $t(9)=5.89$, $p<0.0001$.

The activation maps for individual patients generated from singlesubject analyses are presented in Fig. 5, in the interests of assessing the predictability of the findings across this group of patients. These analyses show that all patients who reported being left-handed are right-hemisphere dominant for language. These results are detailed in Table 3.

\section{Discussion}

The findings from this collection of fMRI studies support the use of the language paradigms applied in our Epilepsy Unit. While activation patterns are widely distributed through these patients' dominant hemispheres, areas crucial for expressive and receptive language function can be isolated and reliably mapped. The findings are clearly meaningful at both the individual and group level.

The value of fMRI for determining hemispheric dominance, or lateralising language, is self-evident. In single-subject analyses at our centre, a voxel count is typically conducted in each hemisphere using specified threshold settings in order to adhere to standardised criteria. Areas critical to language function are localised by assessing activation patterns in pre-defined anatomical regions, namely in expressive and receptive language areas. Activation in these regions is also considered across both language tasks, to assess reproducibility of signal change. Receptive language paradigms are reported to frequently produce bilateral signal increase in posterior superior temporal cortex. ${ }^{[16]}$
Five of the 22 patients in this series of studies showed bilateral signal changes in receptive cortex in response to the passive listening task. This provides important information about the extent of functional reserve in the contralateral hemisphere that would not be acquired from Wada testing.

Expressive language cortex in the dominant inferior, posterior frontal lobe and receptive cortex in the dominant superior posterior temporal lobe, together with the white matter pathway connecting these two regions, the arcuate fasciculus, is commonly cited as the neural substrate of language. It is generally agreed, however, that the anatomical representation of language is not this simple and that language involves a number of discrete and interacting cognitive processes that are subserved by a distributed network of additional brain regions. ${ }^{[18]}$ An additional area critical to confrontation naming, a sub-function of language, lies in the baso-lateral temporal lobe..$^{[19-21]}$ Patients with temporal lobe epilepsy (TLE) often report word-finding difficulties and exhibit below-average performance on measures of confrontation naming. The extent of the neocortical (lateral) resection in patients undergoing dominant temporal lobe surgery determines the probability of new (in those without pre-existing dysnomia) or additional word-finding difficulties after surgery. Hence, identifying the neural correlate of naming is important in presurgical TLE patients, and we have added the auditory naming task ${ }^{[22]}$ to our language-mapping paradigm to identify regions of activation that may be unique to descriptive naming.

False-positive and false-negative results from BOLD contrast fMRI for defining language representation must be considered. Clusters of BOLD signal changes vary in size, depending on the thresholds used to display the data. In addition, the region of maximal BOLD activation does not always correlate with disruption to language as defined by direct cortical stimulation. ${ }^{[16]}$ Since fMRI identifies areas involved in language and ECS effectively identifies those areas critical for language, functional imaging may be best utilised as one component in a broader language-mapping protocol.

Accordingly, the results from the routine language fMRI in our unit are used as a step in a multi-tiered approach to localise language in pre-surgical epilepsy patients. These results, considered in the context of results from neuropsychological testing, are subsequently used to inform and guide extra-operative and intra-operative ECS. The benefit of having fMRI results to tailor and execute ECS testing in a more expedient and focused manner is self-evident. On the basis of our findings to date, the fMRI paradigm described above has been clinically useful for defining cortical representation of motor and language function.

\section{References}

1. Wiebe S, Blume WT, Girvin JP, Eliasziw M. A randomized, controlled trial of surgery for temporal-lobe epilepsy. N Engl J Med 2001;345(5):311-318. [http://www.nejm.org/doi/full/10.1056/NEJM200108023450501]

2. Téllez-Zenteno JF, Dhar R, Wiebe S. Long-term seizure outcomes following epilepsy surgery: A systematic review and meta-analysis. Brain 2005;128(5):1188-1198. [http://dx.doi.org/10.1093/brain/awh449]

3. Wada J, Rasmussen T. Intracarotid injection of sodium amytal for the lateralization of cerebral speech dominance: Experimental and clinical observations. J Neurosurg 1960;17:266-282.

4. Ammerman JM, Caputy AJ, Potolicchio SJ. Endovascular ablation of a temporal lobe epileptogenic focus: A complication of Wada testing. Acta Neurol Scand 2005;112(3):189-191. [http://dx.doi. org /10.1111/j.1600-0404.2005.00459.x]

Gaillard WD, Balsamo L, Xu B, et al. fMRI language task panel improves determination of language dominance. Neurology 2004;63(8):1403-1408.

6. Swanson SJ, Sabsevitz DS, Hammeke T, Binder JR. Functional magnetic resonance imaging of language 6. Swanson S], Sabsevitz DS, Hammeke T, Binder JR. Functional magnetic resonance imaging of langua
in epilepsy. Neuropsychol Rev 2007;17(4):491-504. [http://dx.doi.org/10.1007/s1 1065-007-9050-x]

7. Woermann FG, Jokeit H, Luerding R, Freitag H, Schulz R, Guertler S, et al. Language lateralization by Wada test and fMRI in 100 patients with epilepsy. Neurology 2003;61(5):699-701.
W.

6. Dada test and fMRI in 100 patients with epilepsy. Neurology 2003;61(5):699-701.
8ym RJ, Burns J, Freeman K, Lipton ML. Is functional MR imaging assessment of hemispheric languange dominance as good as the Wada test?: A meta-analysis. Radiology. 2011;261(2):446-55. [http://dx.doi.org/10.1148/radiol.11101344]

9. Giussani C, Roux F-E, Ojemann J, Sganzerla EP, Pirillo D, Papagno C. Is preoperative functional magnetic resonance imaging reliable for language areas mapping in brain tumor surgery? Review of language functional magnetic resonance imaging and direct cortical stimulation correlation studies. Neurosurgery 2010;66(1):113-120. [http://dx.doi.org/10.1227/01.NEU.0000360392.15450.C9] 10. Faro SH, Mohammed FB. Functional MRI: Basic principles and clinical applications. New York: Springer, 2006. 


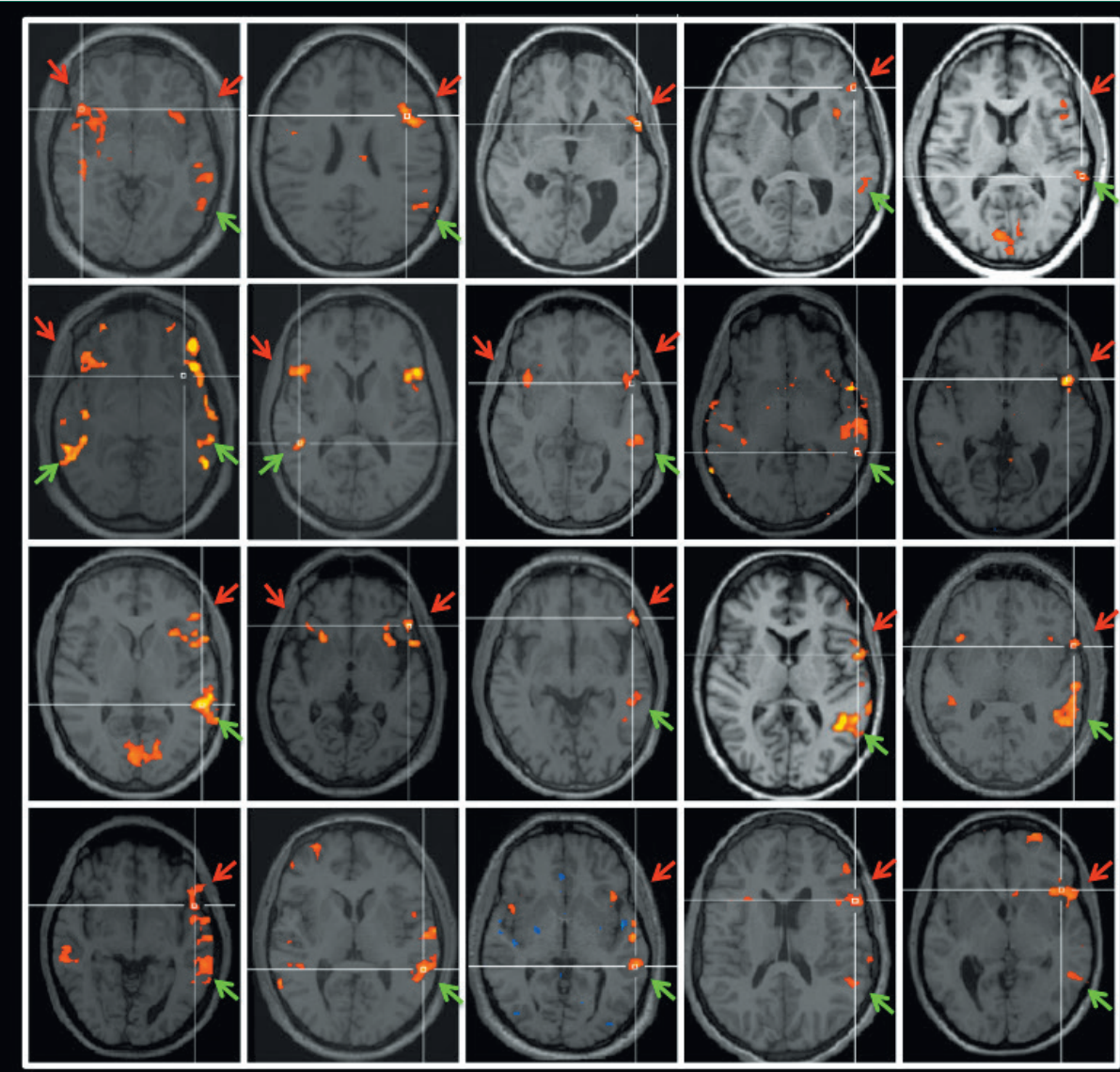

Right handed $=20$

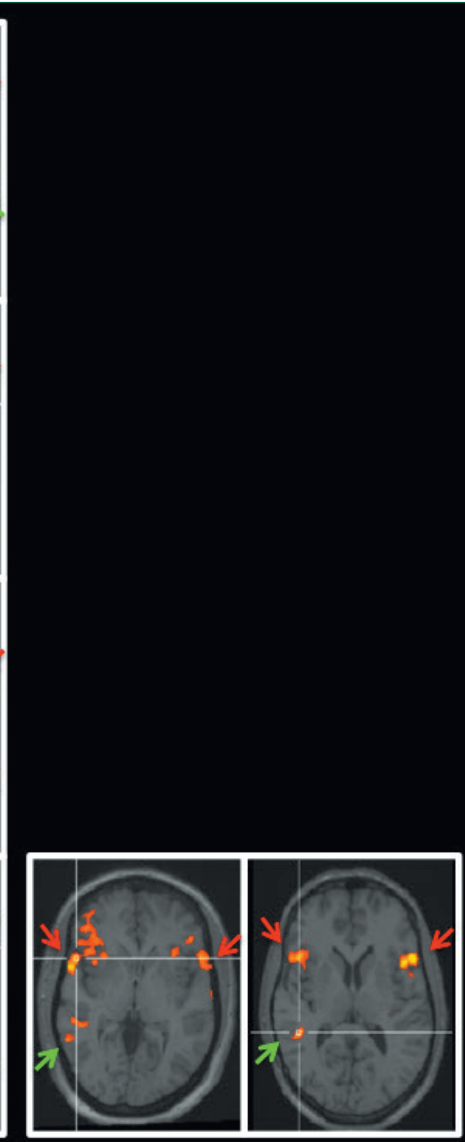

Left handed $=20$

Fig. 5. BOLD signal increases produced in right-and left-handed patients in single-subject analyses during the verb generation task. Note: Red arrows point to expressive language cortex and green arrows to receptive language cortex.

11. Smits M, Visch-Brink E, Schraa-Tam CK, Koudstaal PJ, Van der Lugt A. Functional MR imaging of language processing: An overview of easy-to-implement paradigms for patient care and clinical research. Radiographics 2006;26:S145-S158. [http://dx.doi.org/10.1148/rg.26si065507]

12. Abbott DF, Waites AB, Lillywhite LM, Jackson GD fMRI assessment of language lateralization: An objective approach. Neuroimage 2010;50(4):1446-1455. [http://dx.doi.org/10.1016/j.neuroimage.2010.01.059]

13. Suarez RO, Whalen S, Nelson AP, Tie Y, Meadows M-E, Radmanesh A, et al. Threshold-independent functional MRI determination of language dominance: A validation study against clinical gold standards. Epilepsy Behav 2009;16(2):288-297. [http://dx.doi.org/10.1016/j.yebeh.2009.07.034]

14. Adcock JE, Wise RG, Oxbury JM, Oxbury SM, Matthews PM. Quantitative fMRI assessment of the differences in lateralization of language-related brain activation in patients with temporal lobe epilepsy. Neuroimage 2003;18(2):423-438.

15. McGraw P, Mathews VP, Wang Y, Phillips MD. Approach to functional magnetic resonance imaging of language based on models of language organization. Neuroimaging Clin N Am 2001;11(2):343-353.

16. Duncan JS. Imaging in the surgical treatment of epilepsy. Nat Rev Neurol 2010;6(10):537-550. [http:// dx.doi.org/10.1038/nrneurol.2010]

17. Oldfield RC. The assessment and analysis of handedness: The Edinburgh inventory. Neuropsychologia 1971;9(1):97-113.
18. Hickok G. The functional neuroanatomy of language. Phys Life Rev. 2009;6(3):121-143. [http://dx.doi. org/10.1016/j.plrev.2009.06.001]

19. Damasio H, Tranel D, Grabowski T, Adolphs R, Damasio A. Neural systems behind word and concept retrieval. Cognition 2004;92(1-2):179-229. [http://dx.doi.org/10.1016/j.cognition.2002.07.001]

20. Tranel D, Grabowski TJ, Lyon J, Damasio H. Naming the same entities from visual or from auditory stimulation engages similar regions of left inferotemporal cortices. J Cogn Neurosci 2005;17(8):12931305. [http://dx.doi.org/10.1162/0898929055002508]

21. DeLeon J, Gottesman RF, Kleinman JT, Newhart M, Davis C, Heidler-Gary J, et al. Neural regions essential for distinct cognitive processes underlying picture naming. Brain 2007;130(Pt 5):1408-1422. [http://dx.doi.org/10.1093/brain/awm011]

22. Hamberger MJ, Seidel WT. Auditory and visual naming tests: Normative and patient data for accuracy response time, and tip-of-the-tongue. J Int Neuropsychol Soc 2003;9(3):479-489.

Accepted 24 December 2012. 